\title{
Is salt intake reduction a universal intervention for both normotensive and hypertensive people: a case from Iran STEPS survey 2016
}

\author{
Ali Gholami ${ }^{1,2,3} \cdot$ Shahabeddin Rezaei $^{4,5} \cdot$ Leila Moosavi Jahromi $^{6} \cdot$ Hamid Reza Baradaran $^{3,7} \cdot$ Ali Ghanbari $^{4,8}$. \\ Shirin Djalalinia ${ }^{4,9} \cdot$ Nazila Rezaei $^{4} \cdot$ Shohreh Naderimagham ${ }^{4} \cdot$ Mitra Modirian $^{4} \cdot$ Negar Mahmoudi $^{4}$. \\ Zohreh Mahmoudi ${ }^{4,10} \cdot$ Mohammad Javad Hajipour $^{4,11} \cdot$ Ahmad kousha $^{12} \cdot$ Siamak Mirab Samiee $^{13}$. \\ Farshad Farzadfar ${ }^{4,10}$
}

Received: 7 March 2019 / Accepted: 20 November 2019 / Published online: 4 December 2019

(c) Springer-Verlag GmbH Germany, part of Springer Nature 2019

\begin{abstract}
Purpose There is a direct association between salt intake and blood pressure (BP), one of the main risk factors for CVDs. However, yet there has been a debate that how strong is this association in people with and without hypertension. This study was conducted to evaluate the magnitude of the association between salt intake and BP in hypertensive and normotensive population among a nationally representative population.

Methods The study was conducted on a nationally representative sample of 18,635 Iranian adults aged 25 years and older who participated in the STEPS survey 2016 and provided urine sample. Salt intake was estimated through spot urine sample and Tanaka equation. Multiple linear regression model in survey data analysis was used to assess the independent effect of salt intake on BP.

Results After adjusting for covariates, there was a significant association between salt intake and SBP in hypertensive $(p<0.001)$ and normotensive people $(p<0.001)$. In hypertensive people, with $1 \mathrm{~g}$ of increase in salt intake, the SBP and DBP increased $0.37 \mathrm{mmHg}$ and $0.07 \mathrm{mmHg}$, respectively. Whereas in normotensive people, with $1 \mathrm{~g}$ of increase in salt intake, the SBP and DBP increased $0.26 \mathrm{mmHg}$ and $0.05 \mathrm{mmHg}$, respectively. Moreover, there was a significant trend toward an increase of SBP across salt intake quartiles in both hypertensive $(p<0.001)$ and normotensive people $(p=0.002)$, though the slope was steeper in hypertensive than in normotensive people.

Conclusions The present study demonstrated that salt intake significantly increased SBP in both hypertensive and normotensive people, though the magnitude of this increase was greater in hypertensive people as compared with normotensive people.
\end{abstract}

Keywords Salt $\cdot$ Blood pressure $\cdot$ Hypertensive $\cdot$ Normotensive $\cdot$ Iran

\section{Introduction}

Excess salt intake is associated with the development of several non-communicable diseases, including cardiovascular diseases (CVDs), the leading cause of death [1] and stomach cancer, the third leading cause of cancer death worldwide [2, $3]$. Diet high in sodium was responsible for $20.32 \%$ hypertensive heart disease death, $13.47 \%$ stroke death, $11.58 \%$ ischemic heart diseases death, and $9.83 \%$ stomach cancer death worldwide [4]. Excess salt intake exerts its detrimental

Farshad Farzadfar

f-farzadfar@tums.ac.ir

Extended author information available on the last page of the article effect directly or through increasing the blood pressure (BP) [3].

Hypertension (HTN) is one of the main risk factors for CVDs. It has been estimated that HTN is responsible for more than half of deaths from stroke and coronary heart disease in adults worldwide [4]. The role of excess salt intake in increasing BP has been confirmed in the previous epidemiological and clinical studies [5]. However, debates exist over the relationship of salt intake and its effect on BP in the general population $[6,7]$. These debates focus on findings achieved from some clinical trials with the controlled circumstance that it might not be simply generalized to general populations. In this setting, a high-quality observational study has revealed only a weak association between sodium intake and BP in the general population [8]. 
It was indicated that salt restriction interventions had a lower effect on BP in normotensive people, compared to hypertensive people [4]. In addition, the extreme salt reduction could lead to unfavorable effect in the general population [9]. Therefore, knowing the differences between the association of salt intake and BP in both hypertensive and normotensive people could assist health policymakers to consider this difference in the salt reduction policies. To date, there are limited data on the relationship between salt intake and BP in hypertensive and normotensive people [10]. Therefore, the objective of the present study was to evaluate the magnitude of the association between salt intake and BP in hypertensive and normotensive people.

\section{Materials and methods}

\section{Design and sample}

This study was conducted in the framework of STEPwise approach in surveillance of non-communicable diseases (STEPS) survey in Iran, during 2016. A detailed description of the study design is provided in the study protocol of the study [11]. In brief, the study was a national large-scale cross-sectional survey in that individuals aged 18 years old and above were recruited from urban and rural areas of all provinces of Iran (except Qom province) using stratified random cluster sampling. The STEPS consists of three steps, including (1) questionnaire-based assessment, (2) physical measurement, and (3) biochemical measurements [12]. In Iran, STEPS 2016 among the 31,050 recruited participants, 30,541 participants completed the first step (questionnaire) and 30,042 participants completed the second step (physical measurement). The third step (biochemical measurement) was merely performed in participants aged 25 years and above. A total of 2803 out of 30,541 participants were in the age category of 18-25 years and did not invite to participate in the third step. Therefore, 27,738 subjects were eligible and invited for biochemical measurement; however, 19,778 subjects participated in the third step (response rate $=71.3 \%$ ). The participants who did not take part in the third step (7960 subjects) were defined as nonresponsive. We considered non-response bias weight in our survey analysis. Among the participants who completed the biochemical measurements, 18,635 participants had a proper urine sodium measurement and were included in this study [11]. The remaining (1143 subjects) were considered as missing data.

\section{Measures}

Salt intake and BP were used as independent and dependent factors in this study. Some other factors were used as covariates in relationship between salt intake and BP, including: age, sex, educational level, marital status, local residence, tobacco use, alcohol consumption, fruit intake, vegetable intake, weight status, low physical activity, wealth index, province, having diabetes, total cholesterol, hypertension awareness, and treatment history for hypertension.

\section{Measurement and definition of dependent, independent and covariates factors}

BP $(\mathrm{mmHg})$ was measured thrice using a digital sphygmomanometer (Beurer BM20, Beurer GmbH, Ulm, Germany) after a rest of $15 \mathrm{~min}$ in sitting position. Hypertension was defined (definition 1) as having any of the following items: (1) $\mathrm{BP} \geq 130 / 80 \mathrm{mmHg}$ [13]; (2) use of anti-hypertensive medications; (3) self-report of hypertension. We also defined (definition 2) hypertension based on same criteria except that $\mathrm{BP} \geq 140 / 90 \mathrm{mmHg}$ [14] instead of $\mathrm{BP} \geq 130 / 80 \mathrm{mmHg}$ and we analyzed the association of salt intake and BP according to these two definitions. To estimate salt intake, urinary spot samples (collected in the morning) were taken in STEPS survey. The Tanaka equation [15] was used to estimate $24 \mathrm{~h}$ urinary sodium. Then, the obtained values were multiplied by 2.54 and divided by 1000 to get salt intake ( $\mathrm{g} /$ day) for each person [16].

The data of age, sex, educational level, marital status, local residence, tobacco use, alcohol consumption, fruit intake, vegetable intake, weight status, low physical activity, assets items, hypertension awareness and treatment history for hypertension were obtained by trained interviewers. BP, height, and weight measurements were performed by trained health personnel. Total cholesterol and fasting blood sugar were measured from collected blood samples. Educational level was categorized into three categories, including less than high-school graduate, high-school graduate, and some college or more. Use of tobacco and alcohol consumption was defined as current user, former user, and never user. Consumption of fruit was categorized as more than 2 servings/day; 2 servings/day; 1 serving/day; less than 1 serving/day; or no fruit intake. Consumption of vegetables was categorized as more than 5 servings/day; $4-5$ servings/ day; 3 servings/day; less than 3 servings/day; or no vegetable intake. Total cholesterol was divided into two categories, including $<200 \mathrm{mg} / \mathrm{dl}$ and $\geq 200 \mathrm{mg} / \mathrm{dl}$. Diabetes was defined as having any of the following criteria: fasting blood sugar (FBS) $\geq 126 \mathrm{mg} / \mathrm{dl}$, intake of anti-diabetic medications, or self-reporting of diabetes. Physical activity was assessed by the second version of Global Physical Activity Questionnaire [17]. Easy-to-collect data on a household's ownership (assets items) and principal component analysis were used to calculate the wealth index. Wealth index was used as a proxy to determine the household economic status of the study population. 


\section{Statistical analysis}

The statistical analysis of this study was conducted on 18,631 individuals aged 25 years and older; four of 18,635 individuals did not have blood pressure measurement. For considering the cluster sampling effect, all the analyses were performed using survey analysis. Multiple linear regression model in survey data analysis was used to assess the independent effect of salt intake on BP among hypertensive and normotensive individuals after adjusting for covariates. The study population was divided into four categories (Q1-Q4) according to quartiles of salt intake. Quartiles 1 through 4 were $\leq 7.89,>7.89-9.47 \geq,>9.47-11.09 \geq,>11$ $.09 \mathrm{~g} /$ day. We used the lowest quartile $(\mathrm{Q} 1)$ as the reference group in some analyses. The results presented in the text are related to the value of the definition 1 of hypertension $(B P \geq 130 / 80 \mathrm{mmHg}$ ) and the results of the second definition of hypertension are provided in the Tables $4,5,6$. The results are reported as mean and standard deviation (SD). All the statistical analyses were performed using STATA software version 14 and $p<0.05$ was considered as statistically significant.

\section{Ethical considerations}

The Ethical Committee of National Institute for Medical Research Development (NIMAD) approved the study (Ethical code: IR.NIMAD.REC.1394.032). All the participants were aware of the study's objectives and methods and gave a written informed consent form prior initiation of the study.

\section{Results}

\section{Characteristics of the study population}

The characteristics of the study population are presented in Table 1 . The mean age of the study population was 46.9 (14.1) years and $52.1 \%$ of them were female (Table 1 ). The mean age of the hypertensive and normotensive population was 52.0 (14.5) and 41.6 (12.6) years, respectively. Mean salt intake was 9.7 (2.4) g/day in hypertensive population and 9.3 (2.3) g/day in a normotensive population. The mean SBP of the study population was 137.6 (18.2) in hypertensive population and $113.0(8.9) \mathrm{mmHg}$ in a normotensive population. The mean DBP of the study population was 84.1 (10.3) in hypertensive population and 69.8 (6.6) $\mathrm{mmHg}$ in a normotensive population. Characteristics of the study population across the quartiles of salt intake and hypertension status are shown in Tables 2, 3 .
Association of salt intake and BP in hypertensive population

The results of the unadjusted and adjusted association between salt intake and BP are demonstrated in Table 4 . The significant associations were observed between salt intake and SBP in the unadjusted ( $\beta 0.43$; 95\% CI $0.25-0.60$; $p<0.001)$ and all the adjusted models $(p<0.05)$ (Table 4). Regarding the DBP, a significant association was observed in the unadjusted model $(\beta 0.15$; 95\% CI $0.05-0.25$; $p<0.01$ ); however, after adjusting for all the covariates, the significance of the association was lost ( $\beta 0.07 ; 95 \%$ CI $0.05-0.18 ; p=0.256$ ). As presented in Table 5, in the adjusted models, comparison of each salt quartile with the first quartile resulted in an increase in most of SBP and DBP values; however, the increase was much higher in the fully adjusted models. In this setting, after adjusting for all covariates (model 4), we observed that participants who consumed $>11.09 \mathrm{~g}$ salt per day (quartile 4) had 3.81 (95\% CI 2.00-5.61) $\mathrm{mmHg}$ SBP significantly higher than those who consumed $\leq 7.89 \mathrm{~g} /$ day (quartile 1) (Fig. 1). Moreover, it was observed that the values of SBP and DBP in each model were significantly different across quartiles $(p<0.05)$ (except models 3 and 4 in DBP).

\section{Association of salt intake and BP in normotensive population}

The association between salt intake and $\mathrm{BP}$ in normotensive population $(\mathrm{BP}<130 / 80 \mathrm{mmHg}$ and no use of anti-hypertensive medications and no reporting of hypertension) is shown in Table 4. The salt intake was significantly associated with SBP in the unadjusted ( $\beta 0.41 ; 95 \%$ CI $0.31-0.50 ; p<0.001)$ and all adjusted models $(p<0.05)$. Regarding the DBP, in the unadjusted model, there was a significant association between salt intake and DBP $(\beta$ 0.17; 95\% CI 0.10, 0.24; $p<0.001$ ); while, in the full adjusted model, no significant association was observed ( $\beta 0.05 ; 95 \%$ CI $0.03-0.13$; $p=0.212$ ).

As presented in Table 6, when the models were adjusted for covariates, comparison of each salt quartile with the first quartile resulted in an increase in most of SBP and DBP values. In this context, we observed that participants who consumed $>11.09 \mathrm{~g}$ salt per day (quartile 4) had $1.39 \mathrm{mmHg}$ (95\% CI 0.52-2.26) SBP significantly higher than those who consumed $\leq 7.89 \mathrm{~g} /$ day (quartile 1 ) in the fully adjusted model $(p<0.05)$ (Fig. 1). Also, when the models were adjusted for covariates, it was observed that the increased values of SBP and DBP in each model were significantly different across quartiles (except models 3 and 4 in DBP). 
Table 1 Characteristics of the study population in the salt study and the overall STEPS study

\begin{tabular}{|c|c|c|c|}
\hline \multirow[t]{2}{*}{ Characteristics } & \multirow[t]{2}{*}{ Overall STEPS study $(n=30,541)$} & \multicolumn{2}{|c|}{ Salt study $(n=18,631)$} \\
\hline & & Normotensive $^{\mathrm{a}}$ & Hypertensive $^{\mathrm{b}}$ \\
\hline Number of subjects, no./total no. (\%) & - & $8073 / 18,631(42.5)$ & $10,558 / 18,631(57.5)$ \\
\hline Salt intake & - & $9.3 \pm 2.3$ & $9.7 \pm 2.4$ \\
\hline Age, years (SD) & $46.9 \pm 14.1$ & $41.6 \pm 12.6$ & $52.0 \pm 14.5$ \\
\hline Female sex, no./total no. (\%) & $15,975 / 30,541(52.1)$ & $4386 / 8073(55.4)$ & $5575 / 10,558(53.1)$ \\
\hline \multicolumn{4}{|l|}{ Educational level, no./total no. (\%) } \\
\hline Less than high-school graduate & $17,852 / 29,471(60.0)$ & $4832 / 7974(58.2)$ & $7591 / 10,285(71.5)$ \\
\hline High-school graduate & $6212 / 29,471(22.1)$ & $1654 / 7974(22.6)$ & $1549 / 10,285(16.9)$ \\
\hline Some college or more & $5407 / 29,471(17.9)$ & $1488 / 7974(19.3)$ & $1145 / 10,285(11.7)$ \\
\hline Marital status, no./total no. (\% married) & $23,114 / 29,929(81.1)$ & $6688 / 8041(83.0)$ & $8817 / 10,520(83.7)$ \\
\hline Local residence, no./total no. (\% urban) & $21,493 / 30,541(78.2)$ & $5238 / 8073(69.3)$ & $6873 / 10,558(70.9)$ \\
\hline \multicolumn{4}{|l|}{ Tobacco use, no./total no. (\%) } \\
\hline Never & $23,656 / 29,987(78.8)$ & $6262 / 8051(78.7)$ & $8199 / 10,540(78.5)$ \\
\hline Former & $2115 / 29,987(6.6)$ & $543 / 8051(6.3)$ & $957 / 10,540(8.6)$ \\
\hline Current & $4216 / 29,987(14.6)$ & $1246 / 8051(15.1)$ & $1384 / 10,540(12.8)$ \\
\hline \multicolumn{4}{|l|}{ Alcohol consumption, no./total no. (\%) } \\
\hline Never drank & $27,563 / 29,877(92.2)$ & $7311 / 8039$ (91.5) & $9815 / 10,526(92.9)$ \\
\hline Former drinker & $1155 / 29,877(4.0)$ & $388 / 8039(4.5)$ & $416 / 10,526(4.2)$ \\
\hline Current drinker & $1159 / 29,877(3.8)$ & $340 / 8039(4.0)$ & $295 / 10,526(3.0)$ \\
\hline \multicolumn{4}{|l|}{ Fruits intake, no./total no. (\%) } \\
\hline No intake & $6524 / 29,939(20.5)$ & $1725 / 8030(20.5)$ & $2435 / 10,533(21.7)$ \\
\hline$<1$ serving & $6027 / 29,939(20.7)$ & $1553 / 8030(20.4)$ & $2169 / 10,533(21.0)$ \\
\hline 1 serving & $12,203 / 29,939(41.9)$ & $3230 / 8030(40.3)$ & $4174 / 10,533(40.4)$ \\
\hline 2 serving & $3841 / 29,939(12.2)$ & $1119 / 8030(13.6)$ & $1324 / 10,533(12.4)$ \\
\hline$>2$ serving & $1344 / 29,939(4.7)$ & $403 / 8030(5.2)$ & $431 / 10,533(4.4)$ \\
\hline \multicolumn{4}{|l|}{ Vegetables intake, no./total no. (\%) } \\
\hline No intake & $9978 / 29,993(32.7)$ & $2536 / 8052(31.2)$ & $3442 / 10,540(32.6)$ \\
\hline$<3$ serving & $7291 / 29,993(24.1)$ & $2015 / 8052(25.7)$ & $2567 / 10,540(24.1)$ \\
\hline 3 serving & $10,199 / 29,993(34.5)$ & $2809 / 8052(34.5)$ & $3610 / 10,540(34.5)$ \\
\hline $4-5$ serving & $1974 / 29,993(6.7)$ & $558 / 8052(6.8)$ & $709 / 10,540(6.8)$ \\
\hline$>5$ serving & $551 / 29,993(2.0)$ & $134 / 8052(1.8)$ & $212 / 10,540(2.1)$ \\
\hline \multicolumn{4}{|l|}{ Weight status, no./total no. (\%) } \\
\hline Underweight & $1176 / 29,124(2.8)$ & $412 / 8073(4.8)$ & 222/10,558 (1.9) \\
\hline Normal & $10,676 / 29,124(34.1)$ & $3383 / 8073(40.2)$ & $2752 / 10,558(26.1)$ \\
\hline Overweight & $10,659 / 29,124(38.5)$ & 2863/8073 (36.8) & $4288 / 10,558(40.8)$ \\
\hline Obese & $6613 / 29,124(24.5)$ & $1415 / 8073(18.2)$ & $3296 / 10,558(31.3)$ \\
\hline Low activity (\%) & $15,157 / 26,965(58.5)$ & $3828 / 7088(55.3)$ & $5329 / 9519(57.1)$ \\
\hline \multicolumn{4}{|l|}{ Wealth index, no./total no. (\%) } \\
\hline Poorest & $5902 / 29,310(18.3)$ & $1556 / 7964(18.4)$ & $2326 / 10,438(20.5)$ \\
\hline Poor & $5864 / 29,310(19.1)$ & $1566 / 7964(19.0)$ & 2194/10,438 (20.9) \\
\hline Moderate & $5863 / 29,310(20.3)$ & $1632 / 7964(21.3)$ & $2118 / 10,438(20.5)$ \\
\hline Rich & $5851 / 29,310(21.6)$ & 1629/7964 (20.6) & $1960 / 10,438(19.4)$ \\
\hline Richest & $5830 / 29,310(20.7)$ & 1581/7964 (20.6) & $1840 / 10,438(18.8)$ \\
\hline Diabetes, no./total no. (\%) & 2011/20,113 (10.9) & $369 / 7962(5.2)$ & $1530 / 10,416(15.1)$ \\
\hline Total cholesterol ( $\geq 200 \mathrm{mg} / \mathrm{dl})$, no./total no. $(\%)$ & $3143 / 20,231(16.0)$ & $840 / 8007(11.0)$ & $2017 / 10,479(19.3)$ \\
\hline Hypertension awareness, no./total no. (\%) & $6296 / 15,933(39.1)$ & - & $4480 / 10,557(41.4)$ \\
\hline Treatment history for hypertension, no. (\%) & $3230 / 14,380(22.6)$ & - & 2310/9492 (23.9) \\
\hline $\mathrm{SBP}, \mathrm{mmHg}$ & $126.7(18.8)$ & $113.0(8.9)$ & $137.6(18.2)$ \\
\hline $\mathrm{DBP}, \mathrm{mmHg}$ & $78.0(11.3)$ & $69.8(6.6)$ & $84.1(10.3)$ \\
\hline
\end{tabular}


Table 1 (continued)

\begin{tabular}{llll}
\hline Characteristics & Overall STEPS study $(n=30,541)$ & \multicolumn{3}{l}{ Salt study $(n=18,631)$} \\
\cline { 3 - 4 } & & Normotensive $^{\mathrm{a}}$ & Hypertensive $^{\mathrm{b}}$ \\
\hline Stroke history in the last year, no./total no. (\%) & $215 / 29,980(0.7)$ & $21 / 8059(0.2)$ & $110 / 10,549(1.0)$ \\
Myocardial infraction, no./total no. (\%) & $441 / 29,968(1.7)$ & $50 / 8058(0.6)$ & $254 / 10,550(2.3)$ \\
Statin medication, no./total no. (\%) & $2259 / 29,980(8.5)$ & $285 / 8058(3.5)$ & $1296 / 10,545(12.1)$ \\
Aspirin medication, no./total no. (\%) & $3133 / 29,957(11.8)$ & $434 / 8054(5.4)$ & $1784 / 10,541(16.9)$ \\
\hline
\end{tabular}

$S D$ standard deviation, $S B P$ systolic blood pressure, $D B P$ diastolic blood pressure

${ }^{a} \mathrm{BP}<130 / 80 \mathrm{mmHg}$ and no use of anti-hypertensive medications and not having history of hypertension

${ }^{b} \mathrm{BP} \geq 130 / 80 \mathrm{mmHg}$ or use of anti-hypertensive medications or self-report of hypertension

\section{Association of salt intake and BP in total population}

After adjusting for all covariates, including salt intake and hypertension status interaction, we found that participants who were in the quartile 2, 3, and 4 had $0.52 \mathrm{mmHg}(95 \%$ CI $0.43-1.47 ; p=0.285), 0.87 \mathrm{mmHg}(95 \%$ CI $0.18-1.92$; $p=0.105)$, and $1.53 \mathrm{mmHg}(95 \%$ CI $0.53-2.53 ; p=0.003$ ) SBP higher than those who were in quartile 1, respectively.

Furthermore, we observed that participants who were in the quartile 2,3 , and 4 had $0.36 \mathrm{mmHg}(95 \%$ CI $0.29-1.02$; $p=0.273), 0.17 \mathrm{mmHg}$ (95\% CI 0.48-0.83; $p=0.603)$, and $0.80 \mathrm{mmHg}$ (95\% CI 0.13-1.47; $p=0.019)$ DBP higher than those who were in quartile 1 , respectively.

\section{Discussion}

In this nationally representative study of Iranian adults, it was found that after adjusting for covariates, there was a significant positive association between salt intake and SBP in hypertensive and normotensive participants. On the contrary, no significant association was observed between salt intake and DBP in both groups.

The association of salt intake and BP was stronger in hypertensive than in normotensive people. For instance, in hypertensive people, after adjusting for all covariates, we observed that with $1 \mathrm{~g}$ of increase in salt intake, the SBP and DBP increased $0.37 \mathrm{mmHg}$ and $0.07 \mathrm{mmHg}$, respectively. Whereas, in the normotensive people, with $1 \mathrm{~g}$ of increase in salt intake, the SBP and DBP increased $0.26 \mathrm{mmHg}$ and $0.05 \mathrm{mmHg}$, respectively. Therefore, the association of salt intake and systolic and diastolic BP in hypertensive people was more than 1.4 times higher than that in normotensive people. The same pattern was observed when the definition 2 of hypertension was included in the analysis.

The findings of the present study are consistent with the findings of the Mente and colleagues' study. Mente and colleagues used the population urban and rural epidemiological (PURE) study's data to find the association between the urinary sodium and potassium excretion and BP. The data of 102,216 adults from 18 countries were analyzed. They used urine specimen and Kawasaki equation to estimate the $24 \mathrm{~h}$ urinary excretion of sodium. They indicated that estimated sodium excretion was more strongly associated with increased BP in individuals with HTN than in those with normal BP. For instance, in hypertensive people, with $1 \mathrm{~g}$ of increase in sodium excretion, the SBP increased $2.49 \mathrm{mmHg}$, while in normotensive people, the SBP increased $1.30 \mathrm{mmHg}(p<0.001)$ [10]. One of the possible reasons for the larger effect of salt intake on hypertensive individuals could be related to differences in salt sensitivity between hypertensive and normotensive people. Hypertensive people have almost two times higher salt sensitivity than normotensive people $[4,18]$.

In this study, it was found that SBP and DBP were greater in the highest quartile of salt intake, when compared with the lowest quartile in both hypertensive and normotensive people. Similarly, in the PURE study, participants who had a higher sodium excretion had a greater change in systolic and diastolic BP than in participants who had a lower sodium excretion. In the PURE study, the sodium excretion was divided into three categories, $<3 \mathrm{~g} / \mathrm{day}, 3-5 \mathrm{~g} /$ day, and $>5 \mathrm{~g} /$ day. They observed that changes in SBP and DBP were positively greater in participants who had a sodium excretion at the level of $>5 \mathrm{~g} /$ day than participants who had a sodium excretion at the level of 3-5 g/day or less than $3 \mathrm{~g} /$ day [10].

The results of the present study are in contrary to the Sharma and colleagues' study. They investigated the association between dietary sodium and potassium intake and BP levels in 6985 US adults with no prior history of hypertension. Sharma and colleagues divided the sodium intake into four quartiles and assessed the association of sodium intake and elevated BP across them. After adjusting for covariates, they observed that there was no significant association between sodium intake and risk of elevated BP [19]. There are several differences between the Sharma and colleagues' study with our study. First, Sharma and colleagues calculated the odds ratio of BP (>130/80 and $>140 / 90 \mathrm{mmHg}$ ) and salt intake. They did not analyze the odds ratio of SBP and DBP with salt intake autonomously. As shown in the 
Table 2 Baseline characteristics of hypertensive ${ }^{a}$ study population across quartiles of salt intake

\begin{tabular}{|c|c|c|c|c|c|}
\hline \multirow[t]{2}{*}{ Characteristics } & \multicolumn{4}{|c|}{ Salt intake (g/day) quartiles } & \multirow[t]{2}{*}{$p$ value } \\
\hline & $\leq 7.89$ & $>7.89-9.47 \geq$ & $>9.47-11.09 \geq$ & $>11.09$ & \\
\hline Total population & 2479 & 2540 & 2657 & 2882 & - \\
\hline Mean age, years (SD) & $51.2 \pm 14.9$ & $51.7 \pm 14.4$ & $52.6 \pm 14.3$ & $52.5 \pm 14.5$ & 0.035 \\
\hline Gender ( $\%$ female $)$ & 59.0 & 54.7 & 49.8 & 49.4 & $<0.001$ \\
\hline \multicolumn{6}{|l|}{ Educational level (\%) } \\
\hline Less than high-school graduate & 68.7 & 68.1 & 71.7 & 76.8 & \multirow[t]{3}{*}{$<0.001$} \\
\hline High-school graduate & 18.4 & 19.2 & 15.9 & 14.3 & \\
\hline Some college or more & 12.9 & 12.7 & 12.4 & 8.9 & \\
\hline Marital status (\% married) & 80.2 & 83.4 & 85.8 & 85.2 & $<0.001$ \\
\hline Local residence (\% urban) & 71.6 & 73.4 & 70.1 & 68.8 & 0.005 \\
\hline \multicolumn{6}{|l|}{ Tobacco use $(\%)$} \\
\hline Never & 77.4 & 78.3 & 78.7 & 79.6 & \multirow[t]{3}{*}{$<0.001$} \\
\hline Former & 7.3 & 8.2 & 8.7 & 10.1 & \\
\hline Current & 15.3 & 13.5 & 12.6 & 10.3 & \\
\hline \multicolumn{6}{|l|}{ Alcohol consumption (\%) } \\
\hline Never drank & 92.6 & 92.8 & 93.0 & 93.0 & \multirow[t]{3}{*}{0.843} \\
\hline Former drinker & 4.0 & 4.1 & 4.4 & 4.2 & \\
\hline Current drinker & 3.4 & 3.1 & 2.6 & 2.8 & \\
\hline \multicolumn{6}{|l|}{ Fruits intake (\%) } \\
\hline No intake & 22.8 & 21.7 & 20.7 & 21.6 & \multirow[t]{5}{*}{0.153} \\
\hline$<1$ serving & 22.3 & 21.1 & 20.5 & 20.5 & \\
\hline 1 serving & 38.9 & 41.8 & 40.0 & 41.0 & \\
\hline 2 serving & 12.2 & 11.6 & 14.1 & 11.7 & \\
\hline$>2$ serving & 3.8 & 3.8 & 4.7 & 5.2 & \\
\hline \multicolumn{6}{|l|}{ Vegetables intake (\%) } \\
\hline No intake & 34.3 & 33.2 & 31.8 & 31.2 & \multirow[t]{5}{*}{0.555} \\
\hline$<3$ serving & 23.3 & 24.0 & 25.6 & 23.3 & \\
\hline 3 serving & 33.2 & 34.3 & 34.3 & 35.9 & \\
\hline 4-5 serving & 7.1 & 6.6 & 6.1 & 7.3 & \\
\hline$>5$ serving & 2.1 & 1.9 & 2.2 & 2.3 & \\
\hline \multicolumn{6}{|l|}{ Weight status, no./total no. (\%) } \\
\hline Underweight & 3.4 & 2.0 & 1.3 & 0.9 & \multirow[t]{4}{*}{$<0.001$} \\
\hline Normal & 30.1 & 27.3 & 27.2 & 20.2 & \\
\hline Overweight & 40.5 & 40.9 & 41.5 & 40.1 & \\
\hline Obese & 26.0 & 29.8 & 30.0 & 38.8 & \\
\hline Low activity (\%) & 58.7 & 58.4 & 56.7 & 54.7 & 0.081 \\
\hline \multicolumn{6}{|l|}{ Wealth index (\%) } \\
\hline Poorest & 18.8 & 19.5 & 20.3 & 23.0 & \multirow[t]{5}{*}{0.017} \\
\hline Poor & 20.8 & 21.1 & 20.0 & 21.5 & \\
\hline Moderate & 20.8 & 19.1 & 21.0 & 21.0 & \\
\hline Rich & 19.9 & 19.7 & 20.2 & 18.1 & \\
\hline Richest & 19.7 & 20.6 & 18.5 & 16.4 & \\
\hline Diabetes (\%) & 14.2 & 15.4 & 14.7 & 16.1 & 0.437 \\
\hline Total cholesterol ( $\geq 200 \mathrm{mg} / \mathrm{dl})(\%)$ & 19.9 & 18.5 & 20.0 & 18.7 & 0.512 \\
\hline Hypertension awareness (\%) & 43.4 & 41.2 & 40.4 & 40.6 & 0.253 \\
\hline Treatment history for hypertension (\%) & 26.0 & 22.6 & 22.8 & 24.3 & 0.100 \\
\hline Mean SBP, mmHg & $136.4 \pm 18.1$ & $137.2 \pm 17.8$ & $137.4 \pm 17.8$ & $139.0 \pm 19.1$ & $<0.001$ \\
\hline Mean DBP, mmHg & $83.6 \pm 9.8$ & $84.0 \pm 10.7$ & $84.4 \pm 9.6$ & $84.5 \pm 11.0$ & $<0.001$ \\
\hline
\end{tabular}

$B P$ blood pressure, $S B P$ systolic blood pressure, $D B P$ diastolic blood pressure

${ }^{a} \mathrm{BP} \geq 130 / 80 \mathrm{mmHg}$ or use of anti-hypertensive medications or self-report of hypertension 
Table 3 Baseline characteristics of normotensive ${ }^{\mathrm{a}}$ study population across quartiles of salt intake

\begin{tabular}{|c|c|c|c|c|c|}
\hline \multirow[t]{2}{*}{ Characteristics } & \multicolumn{4}{|c|}{ Salt intake (g/day) quartiles } & \multirow[t]{2}{*}{$p$ value } \\
\hline & $\leq 7.89$ & $>7.89-9.47 \geq$ & $>9.47-11.09 \geq$ & $>11.09$ & \\
\hline Total population & 2179 & 2118 & 2001 & 1775 & - \\
\hline Mean age, years (SD) & $40.2 \pm 12.5$ & $41.3 \pm 12.3$ & $42.6 \pm 12.5$ & $42.4 \pm 12.8$ & $<0.001$ \\
\hline Gender (\% female) & 61.5 & 58.2 & 51.9 & 48.2 & $<0.001$ \\
\hline \multicolumn{6}{|l|}{ Educational level (\%) } \\
\hline Less than high-school graduate & 54.4 & 56.2 & 57.8 & 66.0 & \multirow[t]{3}{*}{$<0.001$} \\
\hline High-school graduate & 23.4 & 22.4 & 24.3 & 19.7 & \\
\hline Some college or more & 22.2 & 21.4 & 17.9 & 14.3 & \\
\hline Marital status (\% married) & 79.4 & 81.9 & 85.1 & 86.4 & $<0.001$ \\
\hline Local residence (\% urban) & 71.5 & 72.1 & 68.1 & 64.4 & $<0.001$ \\
\hline \multicolumn{6}{|l|}{ Tobacco use (\%) } \\
\hline Never & 77.5 & 79.7 & 79.2 & 78.5 & \multirow[t]{3}{*}{0.701} \\
\hline Former & 6.6 & 5.6 & 6.4 & 6.4 & \\
\hline Current & 15.9 & 14.7 & 14.4 & 15.1 & \\
\hline \multicolumn{6}{|l|}{ Alcohol consumption (\%) } \\
\hline Never drank & 91.4 & 91.7 & 91.5 & 91.3 & \multirow[t]{3}{*}{0.57} \\
\hline Former drinker & 4.1 & 4.8 & 4.6 & 4.5 & \\
\hline Current drinker & 4.5 & 3.5 & 3.9 & 4.2 & \\
\hline \multicolumn{6}{|l|}{ Fruits intake* $(\%)$} \\
\hline No intake & 20.9 & 18.9 & 20.5 & 21.8 & \multirow[t]{5}{*}{0.50} \\
\hline$<1$ serving & 20.7 & 21.5 & 20.7 & 18.3 & \\
\hline 1 serving & 38.8 & 40.7 & 40.1 & 42.1 & \\
\hline 2 serving & 13.7 & 13.8 & 13.6 & 13.1 & \\
\hline$>2$ serving & 5.9 & 5.1 & 5.1 & 4.7 & \\
\hline \multicolumn{6}{|l|}{ Vegetables intake* $(\%)$} \\
\hline No intake & 32.5 & 29.6 & 30.4 & 32.6 & \multirow[t]{5}{*}{0.081} \\
\hline$<3$ serving & 24.8 & 28.0 & 24.3 & 25.8 & \\
\hline 3 serving & 33.9 & 33.5 & 36.4 & 34.3 & \\
\hline $4-5$ serving & 6.5 & 7.6 & 7.1 & 5.9 & \\
\hline$>5$ serving & 2.3 & 1.3 & 1.8 & 1.4 & \\
\hline \multicolumn{6}{|l|}{ Weight status, no./total no. (\%) } \\
\hline Underweight & 7.5 & 4.6 & 4.0 & 2.4 & \multirow[t]{4}{*}{$<0.001$} \\
\hline Normal & 46.0 & 41.5 & 38.4 & 33.3 & \\
\hline Overweight & 33.6 & 36.6 & 38.1 & 39.5 & \\
\hline Obese & 12.9 & 17.3 & 19.5 & 24.8 & \\
\hline Low activity (\%) & 55.8 & 58.3 & 54.1 & 52.0 & 0.014 \\
\hline \multicolumn{6}{|l|}{ Wealth index $(\%)$} \\
\hline Poorest & 17.7 & 17.1 & 18.4 & 21.0 & \multirow[t]{5}{*}{0.269} \\
\hline Poor & 19.1 & 19.5 & 18.0 & 19.6 & \\
\hline Moderate & 20.5 & 21.7 & 21.6 & 21.6 & \\
\hline Rich & 20.8 & 20.4 & 21.4 & 19.5 & \\
\hline Richest & 21.9 & 21.3 & 20.6 & 18.3 & \\
\hline Diabetes (\%) & 4.4 & 4.9 & 4.8 & 6.9 & 0.044 \\
\hline Total cholesterol $(\geq 200 \mathrm{mg} / \mathrm{dl})(\%)$ & 11.1 & 12.4 & 10.3 & 9.8 & 0.178 \\
\hline Mean SBP, mmHg & $112.0 \pm 8.9$ & $112.7 \pm 8.9$ & $113.5 \pm 8.8$ & $114.3 \pm 8.8$ & $<0.001$ \\
\hline Mean DBP, mmHg & $69.3 \pm 6.7$ & $69.7 \pm 6.6$ & $69.9 \pm 6.5$ & $70.3 \pm 6.6$ & $<0.001$ \\
\hline
\end{tabular}

$B P$ blood pressure, $S B P$ systolic blood pressure, $D B P$ diastolic blood pressure

${ }^{\mathrm{a}} \mathrm{BP}<130 / 80 \mathrm{mmHg}$ and no use of anti-hypertensive medications, and not having history of hypertension 
Table 4 Unadjusted and adjusted relationship of salt intake with SBP and DBP in hypertensive and normotensive participants

\begin{tabular}{|c|c|c|c|c|c|c|c|}
\hline & & \multicolumn{3}{|l|}{ SBP } & \multicolumn{3}{|l|}{ DBP } \\
\hline & & $\beta$ & $95 \% \mathrm{CI}$ & $p$ value & $\beta$ & $95 \% \mathrm{CI}$ & $p$ value \\
\hline \multicolumn{8}{|c|}{ (A) Hypertensive subjects } \\
\hline \multirow[t]{5}{*}{$\geq 130 / 80^{\mathrm{a}}(\mathrm{mmHg})$} & Unadjusted & 0.43 & $(0.25,0.60)$ & $<0.001$ & 0.15 & $(0.05,0.25)$ & 0.002 \\
\hline & Adjusted (model 1) & 0.34 & $(0.17,0.51)$ & $<0.001$ & 0.11 & $(0.01,0.20)$ & 0.027 \\
\hline & Adjusted (model 2) & 0.37 & $(0.20,0.54)$ & $<0.001$ & 0.11 & $(0.01,0.20)$ & 0.032 \\
\hline & Adjusted (model 3) & 0.30 & $(0.11,0.49)$ & 0.002 & 0.05 & $(-0.05,0.16)$ & 0.333 \\
\hline & Adjusted (model 4) & 0.37 & $(0.18,0.56)$ & $<0.001$ & 0.07 & $(-0.05,0.18)$ & 0.256 \\
\hline \multirow[t]{5}{*}{$\geq 140 / 90^{\mathrm{b}}(\mathrm{mmHg})$} & Unadjusted & 0.54 & $(0.31,0.78)$ & $<0.001$ & 0.26 & $(0.12,0.40)$ & $<0.001$ \\
\hline & Adjusted (model 1) & 0.51 & $(0.28,0.74)$ & $<0.001$ & 0.22 & $(0.08,0.36)$ & 0.002 \\
\hline & Adjusted (model 2) & 0.53 & $(0.29,0.76)$ & $<0.001$ & 0.21 & $(0.07,0.36)$ & 0.003 \\
\hline & Adjusted (model 3) & 0.48 & $(0.23,0.74)$ & $<0.001$ & 0.16 & $(0.01,0.31)$ & 0.042 \\
\hline & Adjusted (model 4) & 0.47 & $(0.21,0.74)$ & $<0.001$ & 0.15 & $(-0.01,0.31)$ & 0.068 \\
\hline \multicolumn{8}{|c|}{ (B) Normotensive subjects } \\
\hline \multirow[t]{5}{*}{$<130 / 80^{\mathrm{c}}(\mathrm{mmHg})$} & Unadjusted & 0.41 & $(0.31,0.50)$ & $<0.001$ & 0.17 & $(0.10,0.24)$ & $<0.001$ \\
\hline & Adjusted (model 1) & 0.35 & $(0.25,0.45)$ & $<0.001$ & 0.14 & $(0.07,0.22)$ & $<0.001$ \\
\hline & Adjusted (model 2) & 0.32 & $(0.22,0.42)$ & $<0.001$ & 0.13 & $(0.05,0.20)$ & 0.001 \\
\hline & Adjusted (model 3) & 0.24 & $(0.13,0.35)$ & $<0.001$ & 0.04 & $(-0.04,0.12)$ & 0.320 \\
\hline & Adjusted (model 4) & 0.26 & $(0.15,0.37)$ & $<0.001$ & 0.05 & $(-0.03,0.13)$ & 0.212 \\
\hline \multirow[t]{5}{*}{$<140 / 90^{\mathrm{d}}(\mathrm{mmHg})$} & Unadjusted & 0.49 & $(0.40,0.59)$ & $<0.001$ & 0.23 & $(0.16,0.31)$ & $<0.001$ \\
\hline & Adjusted (model 1) & 0.40 & $(0.31,0.50)$ & $<0.001$ & 0.19 & $(0.11,0.26)$ & $<0.001$ \\
\hline & Adjusted (model 2) & 0.38 & $(0.29,0.48)$ & $<0.001$ & 0.17 & $(0.09,0.24)$ & $<0.001$ \\
\hline & Adjusted (model 3) & 0.27 & $(0.17,0.38)$ & $<0.001$ & 0.06 & $(-0.02,0.14)$ & 0.124 \\
\hline & Adjusted (model 4) & 0.29 & $(0.18,0.39)$ & $<0.001$ & 0.07 & $(-0.01,0.16)$ & 0.078 \\
\hline
\end{tabular}

Model 1: adjusted for age, sex, educational level, marital status, and local residence. Model 2: adjusted for age, sex, educational level, marital status, local residence, tobacco use, and alcohol consumption, fruit intake, vegetable intake. Model 3: adjusted for age, sex, educational level, marital status, local residence, tobacco use and alcohol consumption, fruit intake, vegetable intake, weight status, low activity, wealth index ,and province. Model 4: adjusted for age, sex, educational level, marital status, local residence, tobacco use, alcohol consumption, fruit intake, vegetable intake, weight status, low activity, wealth index, province, diabetes, total cholesterol, hypertension awareness (exclusively for hypertensive individuals), treatment history for hypertension (exclusively for hypertensive individuals)

$S B P$ systolic blood pressure, $D B P$ diastolic blood pressure, g/day gram per day

${ }^{a} \mathrm{BP} \geq 130 / 80 \mathrm{mmHg}$ or use of anti-hypertensive medications or self-report of hypertension

${ }^{b} \mathrm{BP} \geq 140 / 90 \mathrm{mmHg}$ or use of anti-hypertensive medications or self-report of hypertension

${ }^{\mathrm{c}} \mathrm{BP}<130 / 80 \mathrm{mmHg}$ and no use of anti-hypertensive medications and not having history of hypertension

${ }^{\mathrm{d}} \mathrm{BP}<140 / 90 \mathrm{mmHg}$ and no use of anti-hypertensive medications and not having history of hypertension present study, we did not find a significant association between salt intake and DBP. Therefore, if they calculated the odds ratio of salt intake and systolic and diastolic BP autonomously, they might find an association between salt intake and SBP or DBP. The second differences could be related to the confounder factors that were used in Sharma and colleagues' study and the present study to find the association between salt intake and BP.

This study had several strengths. First, this study was performed at the national level and the data were representative sample of Iranian population. Second, the association of salt intake was assessed with two classifications of HTN. Third, the relationship between salt intake and BP was assessed after adjusting for confounders that could affect BP, including age, sex, educational level, marital status, local residence, tobacco use, alcohol consumption, fruit intake, vegetable intake, weight status, low activity, wealth index, province, diabetes, total cholesterol, hypertension awareness (exclusively for hypertensive individuals), treatment history for hypertension (exclusively for hypertensive individuals), and interaction between salt intake, total cholesterol, and educational level.

Limitations worth mentioning are the cross-sectional design of the study, which does not allow for causality and method of salt intake measurement. Salt intake was measured by spot urine sample and salt intake estimation formulas, though the gold standard for assessing the salt intake is a measurement of $24 \mathrm{~h}$ urinary excretion of sodium. However, 
Table 5 Unadjusted and adjusted relationship of salt intake with SBP and DBP in hypertensive participants across quartiles of salt intake

\begin{tabular}{|c|c|c|c|c|c|}
\hline & \multicolumn{4}{|c|}{ Salt intake (g/day) quartiles } & \multirow[t]{2}{*}{$p$ for trend } \\
\hline & $\leq 7.89$ & $>7.89-9.47 \geq$ & $>9.47-11.09 \geq$ & $>11.09$ & \\
\hline \multicolumn{6}{|l|}{$\geq 130 / 80^{\mathrm{a}}(\mathrm{mmHg})$} \\
\hline \multicolumn{6}{|l|}{ SBP } \\
\hline Unadjusted & Ref & $0.87(-0.33,2.06)$ & $1.08(-0.14,2.29)^{*}$ & $2.64(1.43,3.85)^{* *}$ & $<0.001$ \\
\hline Adjusted (model 1) & Ref & $0.74(-0.37,1.86)$ & $0.77(-0.36,1.91)$ & $2.17(1.01,3.33)^{* *}$ & $<0.001$ \\
\hline Adjusted (model 2) & Ref & $0.78(-0.34,1.90)$ & $0.85(-0.31,2.00)$ & $2.26(1.09,3.43)^{* *}$ & $<0.001$ \\
\hline Adjusted (model 3) & Ref & $0.72(-0.47,1.91)$ & $0.63(-0.61,1.87)$ & $1.92(0.65,3.19)^{* *}$ & $<0.001$ \\
\hline Adjusted (model 4) & Ref & $1.15(-0.53,2.82)$ & $1.92(0.06,3.79)^{* *}$ & $3.81(2.00,5.61)^{* *}$ & $<0.001$ \\
\hline \multicolumn{6}{|l|}{ DBP } \\
\hline Unadjusted & Ref & $0.36(-0.31,1.04)$ & $0.75(0.13,1.36)^{* *}$ & $0.84(0.18,1.49)^{* *}$ & $<0.001$ \\
\hline Adjusted (model 1) & Ref & $0.25(-0.44,0.93)$ & $0.58(-0.04,1.21)^{*}$ & $0.53(-0.12,1.18)$ & $<0.001$ \\
\hline Adjusted (model 2) & Ref & $0.23(-0.46,0.92)$ & $0.55(-0.08,1.18)^{*}$ & $0.52(-0.14,1.18)$ & 0.002 \\
\hline Adjusted (model 3) & Ref & $0.13(-0.59,0.85)$ & $0.30(-0.38,0.97)$ & $0.20(-0.53,0.92)$ & 0.162 \\
\hline Adjusted (model 4) & Ref & $0.18(-0.83,1.18)$ & $0.55(-0.42,1.53)$ & $1.18(0.16,2.20)^{* *}$ & 0.252 \\
\hline \multicolumn{6}{|l|}{$\geq 140 / 90^{\mathrm{b}}(\mathrm{mmHg})$} \\
\hline \multicolumn{6}{|l|}{ SBP } \\
\hline Unadjusted & Ref & $1.17(-0.42,2.76)$ & $1.62(-0.01,3.26)^{*}$ & $3.28(1.66,4.89)^{* *}$ & $<0.001$ \\
\hline Adjusted (model 1) & Ref & $1.23(-0.33,2.79)$ & $1.44(-0.16,3.05)^{*}$ & $3.09(1.47,4.71)^{* *}$ & $<0.001$ \\
\hline Adjusted (model 2) & Ref & $1.23(-0.32,2.79)$ & $1.50(-0.12,3.11)^{*}$ & $3.15(1.53,4.78)^{* *}$ & $<0.001$ \\
\hline Adjusted (model 3) & Ref & $1.19(-0.44,2.82)$ & $1.27(-0.45,2.99)$ & $3.11(1.36,4.86)^{* *}$ & $<0.001$ \\
\hline Adjusted (model 4) & Ref & $1.23(-1.00,3.45)$ & $2.15(-0.47,4.76)$ & $4.42(1.95,6.89)^{* *}$ & $<0.001$ \\
\hline \multicolumn{6}{|l|}{ DBP } \\
\hline Unadjusted & Ref & $0.77(-0.25,1.79)$ & $1.23(0.30,2.15)^{* *}$ & $1.60(0.63,2.58)^{* *}$ & $<0.001$ \\
\hline Adjusted (model 1) & Ref & $0.59(-0.45,1.62)$ & $1.10(0.16,2.04)^{* *}$ & $1.24(0.27,2.21)^{* *}$ & $<0.001$ \\
\hline Adjusted (model 2) & Ref & $0.55(-0.48,1.58)$ & $1.04(0.10,1.98)^{* *}$ & $1.20(0.23,2.18)^{* *}$ & $<0.001$ \\
\hline Adjusted (model 3) & Ref & $0.38(-0.67,1.43)$ & $0.74(-0.25,1.73)$ & $0.91(-0.15,1.96)^{*}$ & 0.009 \\
\hline Adjusted (model 4) & Ref & $0.25(-1.19,1.69)$ & $0.89(-0.53,2.31)$ & $1.65(0.18,3.13)^{* *}$ & 0.058 \\
\hline
\end{tabular}

Model 1: adjusted for age, sex, educational level, marital status and local residence. Model 2: adjusted for age, sex, educational level, marital status, local residence, tobacco use and alcohol consumption, fruit intake, vegetable intake. Model 3: adjusted for age, sex, educational level, marital status, local residence, tobacco use and alcohol consumption, fruit intake, vegetable intake, weight status, low activity, wealth index and province. Model 4: adjusted for age, sex, educational level, marital status, local residence, tobacco use, alcohol consumption, fruit intake, vegetable intake, weight status, low activity, wealth index, province, diabetes, total cholesterol, hypertension awareness (exclusively for hypertensive individuals), treatment history for hypertension (exclusively for hypertensive individuals) and interaction between salt intake, total cholesterol and educational level

$S B P$ systolic blood pressure, $D B P$ diastolic blood pressure, g/day gram per day

${ }^{a} \mathrm{BP} \geq 130 / 80 \mathrm{mmHg}$ or use of anti-hypertensive medications or self-report of hypertension

${ }^{\mathrm{b}} \mathrm{BP} \geq 140 / 90 \mathrm{mmHg}$ or use of anti-hypertensive medications or self-report of hypertension

$* p<0.1 ; * *<0.05$

in the large-scale studies, measurement of $24 \mathrm{~h}$ urinary sodium is not cost benefit and it could have a negative effect on the collaboration of the participants [20]. According to the World Health Organization "The SHAKE Technical Package for Salt Reduction", spot urine sodium can be considered as an useful alternative to $24 \mathrm{~h}$ urine sodium for estimating the mean population salt intake in the countries that lack the resources or capacity to do $24 \mathrm{~h}$ urine collection properly [21].

For reducing population salt intake, several actions, including monitoring of population salt intake, identifying the main sources of salt in the diet, and designing effective policies for salt reduction, are needed [22]. According to the current survey, the mean salt intake in both hypertensive and normotensive people was greater than the level of recommendation by the World Health Organization (WHO). These values are in accordance with other countries. According to the INTERMAP study, in the UK, the mean salt intake among normotensive, prehypertensive, untreated hypertensive, and treated hypertensive people who were not on reduced salt diet were $8 \mathrm{~g} /$ day, $8.8 \mathrm{~g} /$ day, $8.7 \mathrm{~g} /$ day, and $9 \mathrm{~g} /$ day, respectively. In China, the mean salt intake 


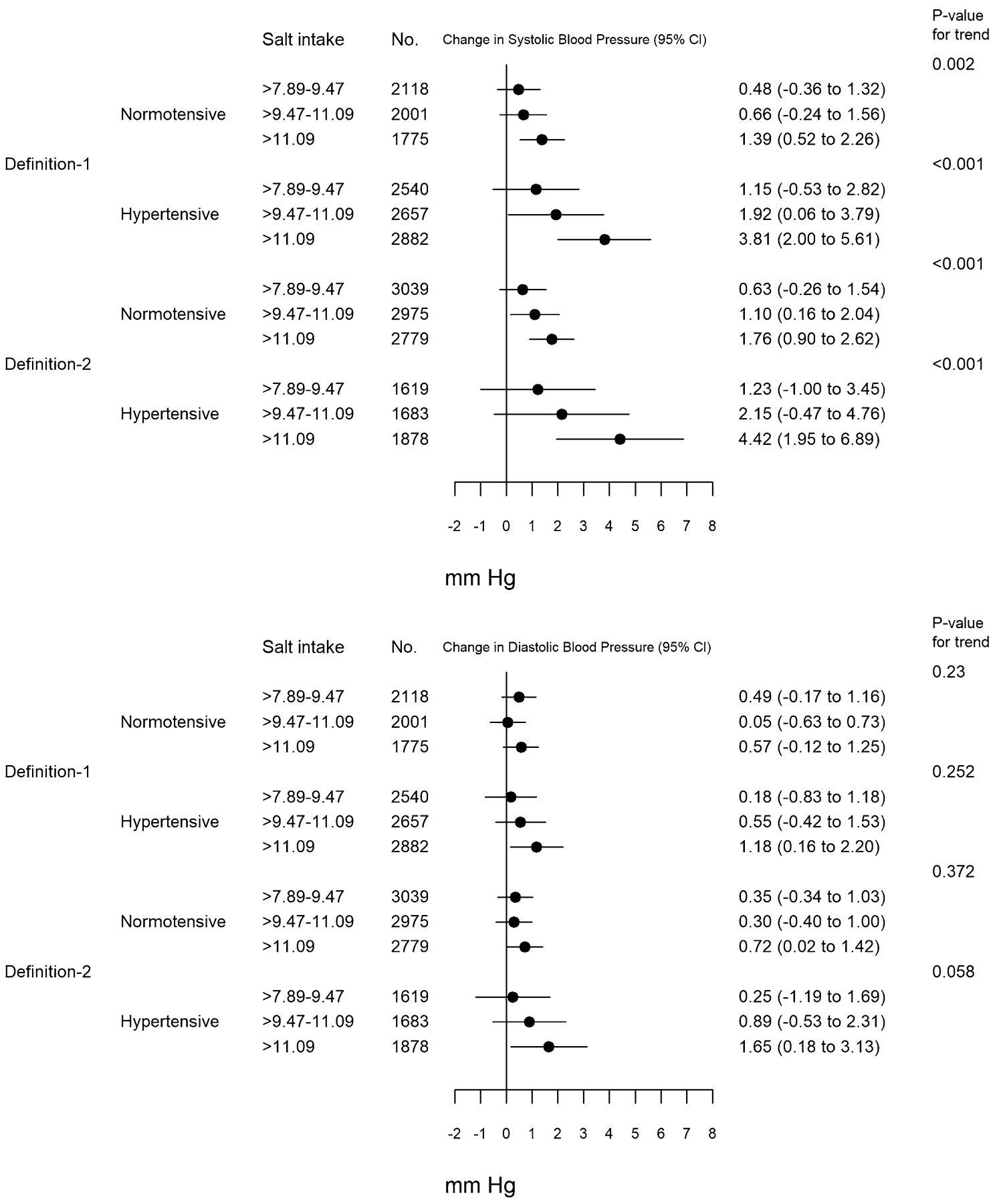

Fig. 1 Forest plot of changes in SBP and DBP of each salt quartile in comparison to the first quartile. Data are based on multivariable linear regression models in survey analysis with adjustment for other studied covariates

in normotensive people was $12.7 \mathrm{~g} /$ day, in prehypertensive people was $13.6 \mathrm{~g} /$ day, in untreated hypertensive people was $14.5 \mathrm{~g} / \mathrm{day}$, and in treated hypertensive people was $15.5 \mathrm{~g} /$ day. The mean salt intake among Japanese normotensive, prehypertensive, untreated hypertensive, and treated hypertensive people was $11.3 \mathrm{~g} /$ day, $12.2 \mathrm{~g} /$ day, $12.2 \mathrm{~g} /$ day, and $11.6 \mathrm{~g} /$ day, respectively. In the USA, the mean salt intake among normotensive, prehypertensive, untreated hypertensive, and treated hypertensive people was $9 \mathrm{~g} / \mathrm{day}, 10 \mathrm{~g} / \mathrm{day}$, $9.8 \mathrm{~g} /$ day and $10.3 \mathrm{~g} /$ day, respectively [23].

In Iran, the main sources of salt intake are bread, cheese, and yogurt drinks [24]. Bread is one of the main sources of salt intake in other countries such as Germany, as well [25]. In European countries, the main sources of salt intake are 
Table 6 Unadjusted and adjusted relationship of salt intake to SBP and DBP in normotensive participants across quartiles of salt intake

\begin{tabular}{|c|c|c|c|c|c|}
\hline & \multicolumn{4}{|c|}{ Salt intake (g/day) quartiles } & \multirow[t]{2}{*}{$p$ for trend } \\
\hline & $\leq 7.89$ & $>7.89-9.47 \geq$ & $>9.47-11.09 \geq$ & $>11.09$ & \\
\hline \multicolumn{6}{|l|}{$<130 / 80^{\mathrm{a}}(\mathrm{mmHg})$} \\
\hline \multicolumn{6}{|l|}{ SBP } \\
\hline Unadjusted & Ref & $0.70(0.08,1.33)^{* *}$ & $1.55(0.91,2.18)^{* *}$ & $2.31(1.67,2.94)^{* *}$ & $<0.001$ \\
\hline Adjusted (model 1) & Ref & $0.61(-0.01,1.22)^{*}$ & $1.26(0.62,1.90)^{* *}$ & $1.93(1.28,2.57)^{* *}$ & $<0.001$ \\
\hline Adjusted (model 2) & Ref & $0.54(-0.07,1.15)^{*}$ & $1.14(0.50,1.79)^{* *}$ & $1.78(1.13,2.43)^{* *}$ & $<0.001$ \\
\hline Adjusted (model 3) & Ref & $0.45(-0.16,1.06)$ & $1.06(0.39,1.73)^{* *}$ & $1.16(0.45,1.87)^{* *}$ & 0.003 \\
\hline Adjusted (model 4) & Ref & $0.48(-0.36,1.32)$ & $0.66(-0.24,1.56)$ & $1.39(0.52,2.26)^{* *}$ & 0.002 \\
\hline \multicolumn{6}{|l|}{$\mathrm{DBP}$} \\
\hline Unadjusted & Ref & $0.48(0.03,0.94)^{* *}$ & $0.59(0.12,1.06)^{* *}$ & $1.00(0.53,1.48)^{* *}$ & $<0.001$ \\
\hline Adjusted (model 1) & Ref & $0.43(-0.01,0.88)^{*}$ & $0.43(-0.04,0.90)^{*}$ & $0.85(0.37,1.33)^{* *}$ & $<0.001$ \\
\hline Adjusted (model 2) & Ref & $0.37(-0.07,0.82)$ & $0.36(-0.11,0.83)$ & $0.74(0.26,1.22)^{* *}$ & 0.002 \\
\hline Adjusted (model 3) & Ref & $0.12(-0.34,0.58)$ & $0.10(-0.40,0.60)$ & $0.15(-0.37,0.67)$ & 0.315 \\
\hline Adjusted (model 4) & Ref & $0.49(-0.17,1.16)$ & $0.05(-0.63,0.73)$ & $0.57(-0.12,1.25)$ & 0.230 \\
\hline \multicolumn{6}{|l|}{$<140 / 90^{\mathrm{b}}(\mathrm{mmHg})$} \\
\hline \multicolumn{6}{|l|}{ SBP } \\
\hline Unadjusted & Ref & $1.03(0.39,1.67)^{* *}$ & $1.89(1.26,2.52)^{* *}$ & $3.02(2.41,3.63)^{* *}$ & $<0.001$ \\
\hline Adjusted (model 1) & Ref & $0.82(0.20,1.44)^{* *}$ & $1.44(0.81,2.08)^{* *}$ & $2.39(1.78,3.00)^{* *}$ & $<0.001$ \\
\hline Adjusted (model 2) & Ref & $0.75(0.14,1.36)^{* *}$ & $1.32(0.69,1.95)^{* *}$ & $2.23(1.62,2.83)^{* *}$ & $<0.001$ \\
\hline Adjusted (model 3) & Ref & $0.60(-0.03,1.23)^{*}$ & $1.17(0.50,1.84)^{* *}$ & $1.6(0.93,2.26)^{* *}$ & $<0.001$ \\
\hline Adjusted (model 4) & Ref & $0.63(-0.26,1.54)$ & $1.10(0.16,2.04)^{* *}$ & $1.76(0.90,2.62)^{* *}$ & $<0.001$ \\
\hline \multicolumn{6}{|l|}{ DBP } \\
\hline Unadjusted & Ref & $0.53(0.05,1.01)^{* *}$ & $0.96(0.48,1.44)^{* *}$ & $1.40(0.92,1.88)^{* *}$ & $<0.001$ \\
\hline Adjusted (model 1) & Ref & $0.40(-0.07,0.87)^{*}$ & $0.67(0.19,1.15)^{* *}$ & $1.05(0.57,1.54)^{* *}$ & $<0.001$ \\
\hline Adjusted (model 2) & Ref & $0.33(-0.14,0.80)$ & $0.58(0.11,1.06)^{* *}$ & $0.94(0.46,1.42)^{* *}$ & $<0.001$ \\
\hline Adjusted (model 3) & Ref & $0.08(-0.40,0.56)$ & $0.28(-0.23,0.78)$ & $0.35(-0.17,0.88)$ & 0.407 \\
\hline Adjusted (model 4) & Ref & $0.35(-0.34,1.03)$ & $0.30(-0.40,1.00)$ & $0.72(0.02,1.42)^{* *}$ & 0.372 \\
\hline
\end{tabular}

Model 1: adjusted for age, sex, educational level, marital status and local residence. Model 2: adjusted for age, sex, educational level, marital status, local residence, tobacco use and alcohol consumption, fruit intake, vegetable intake. Model 3: adjusted for age, sex, educational level, marital status, local residence, tobacco use and alcohol consumption, fruit intake, vegetable intake, weight status, low activity, wealth index and province. Model 4: adjusted for age, sex, educational level, marital status, local residence, tobacco use, alcohol consumption, fruit intake, vegetable intake, weight status, low activity, wealth index, province, diabetes, total cholesterol and interaction between salt intake, total cholesterol and educational level

$S B P$ systolic blood pressure, $D B P$ diastolic blood pressure, g/day gram per day

${ }^{\mathrm{a}} \mathrm{BP}<130 / 80 \mathrm{mmHg}$ and no use of anti-hypertensive medications and not having history of hypertension

${ }^{\mathrm{b}} \mathrm{BP}<140 / 90 \mathrm{mmHg}$ and no use of anti-hypertensive medications and not having history of hypertension

$* p<0.1 ; * * p<0.05$

processed foods, bread, meat, and cheese [25, 26]. In the East Asian countries such as Japan, South Korea, and China, one of the main dietary sources of salt is soy sauce that is used as a seasoning in their foods [26, 27].

To date, several policies have been proposed to reduce the salt intake of populations to the level recommended by WHO or other organizations. Some of these policies have been executed at the population level such as reformulation of the foods, taxation, food labeling, public health campaigns, creating an enabling environment for salt reduction through promotion of healthy food in workplace or school, or advertising controls and some of them have been performed at the individual level such as dietary counseling [26, 28, 29]. The finding of the previous studies sheds light that salt restriction strategies had a different effect on BP in hypertensive and normotensive people [28, 29]. It was revealed that the magnitude of salt restriction effect on BP is higher in hypertensive people than that in normotensive people [4,9]. Furthermore, there is a debate that how much salt reduction is acceptable in the general population. Some of the studies reported that there is a U-shaped association between salt intake and BP that both excess salt intake and severe salt restriction could increase the risk of CVDs [30]. Therefore, policymakers should consider this gap of evidence in the 
salt reduction policies. Moreover, it is suggested that future studies investigate that how much reduction in salt intake is safe and could reduce the risk of CVDs and promote the health status of the general population [30].

\section{Conclusion}

In conclusion, there was a strong association between salt intake and SBP in hypertensive people. In normotensive people, there was a significant association between salt intake and SBP, but not as strong as that in hypertensive people. Based on the findings of the present study and the current evidence, hypertensive people may obtain more benefit from salt restriction policies.

Acknowledgements The authors would like to thank Deputy of Research, Technology, and Deputy of Public Health of the Ministry of Health and Medical Education, Islamic Republic of Iran, and National Institute for Health Research for their collaboration in this study. This work was supported by Iran Ministry of Health and Education and National Institute for Health Research [Grant number: 241,93259].

Author contributions AG, SR, and LMJ had the idea and wrote the primary draft of the manuscript. AG and AG analyzed the data. FF, NR, SD, SN, MM, MJH, AK designed the study. FF, HB revised the manuscript critically. FF, AG, SR contributed in the interpretation of the data. SMS contributed in laboratory measurement designing and interpretation of the laboratory measurement results. NM, ZM contributed in data collection, measurement, and interpretation of the laboratory measurement results.

\section{Compliance with ethical standards}

Conflict of interest The authors declare that there is no conflict of interest.

\section{References}

1. GBD 2016 Causes of Death Collaborators (2017) Global, regional, and national age-sex specific mortality for 264 causes of death, 1980-2016: a systematic analysis for the Global Burden of Disease Study 2016. Lancet 390(10100):1151-1210. https:// doi.org/10.1016/s0140-6736(17)32152-9

2. Ferlay J, Soerjomataram I, Dikshit R, Eser S, Mathers C, Rebelo M, Parkin DM, Forman D, Bray F (2015) Cancer incidence and mortality worldwide: sources, methods and major patterns in GLOBOCAN 2012. Int J Cancer 136(5):E359-E386. https://doi. org/10.1002/ijc.29210

3. He FJ, MacGregor GA (2009) A comprehensive review on salt and health and current experience of worldwide salt reduction programmes. J Hum Hypertens 23(6):363-384. https://doi. org/10.1038/jhh.2008.144

4. Wang M, Moran AE, Liu J, Qi Y, Xie W, Tzong K, Zhao D (2015) A meta-analysis of effect of dietary salt restriction on blood pressure in Chinese adults. Glob Heart 10(4):291-299.e296. https:// doi.org/10.1016/j.gheart.2014.10.009

5. Strazzullo P, D'Elia L, Kandala NB, Cappuccio FP (2009) Salt intake, stroke, and cardiovascular disease: meta-analysis of prospective studies. BMJ 339:b4567. https://doi.org/10.1136/ bmj.b4567

6. Hooper L, Bartlett C, Davey Smith G, Ebrahim S (2002) Systematic review of long term effects of advice to reduce dietary salt in adults. BMJ 325(7365):628

7. Frost CD, Law MR, Wald NJ (1991) By how much does dietary salt reduction lower blood pressure? II-analysis of observational data within populations. BMJ 302(6780):815-818

8. Franco V, Oparil S (2006) Salt sensitivity, a determinant of blood pressure, cardiovascular disease and survival. J Am Coll Nutr 25(3 Suppl):247s-255s

9. Graudal NA, Hubeck-Graudal T, Jurgens G (2017) Effects of low sodium diet versus high sodium diet on blood pressure, renin, aldosterone, catecholamines, cholesterol, and triglyceride. Cochrane Database Syst Rev 4:Cd004022. https://doi. org/10.1002/14651858.cd004022.pub4

10. Mente A, O'Donnell MJ, Rangarajan S, McQueen MJ, Poirier P, Wielgosz A, Morrison H, Li W, Wang X, Di C, Mony P, Devanath A, Rosengren A, Oguz A, Zatonska K, Yusufali AH, Lopez-Jaramillo P, Avezum A, Ismail N, Lanas F, Puoane T, Diaz R, Kelishadi R, Iqbal R, Yusuf R, Chifamba J, Khatib $\mathrm{R}$, Teo K, Yusuf S (2014) Association of urinary sodium and potassium excretion with blood pressure. N Engl J Med 371(7):601-611. https://doi.org/10.1056/NEJMoa1311989

11. Djalalinia S, Modirian M, Sheidaei A, Yoosefi M, Zokaiee H, Damirchilu B, Mahmoudi Z, Mahmoudi N, Hajipour MJ, Peykari N, Rezaei N, Haghshenas R, Mohammadi MH, Delavari A, Gouya MM, Naderimagham S, Kousha A, Moghisi A, Mahdavihezaveh A, Abachizadeh K, Majdzadeh R, Sayyari AA, Malekzadeh R, Larijani B, Farzadfar F (2017) Protocol design for large-scale cross-sectional studies of surveillance of risk factors of non-communicable diseases in Iran: STEPs 2016. Arch Iran Med 20(9):608-616

12. World Health Organization (2003) The WHO STEPwise approach to Surveillance of noncommunicable diseases (STEPS). World Health Organization, Geneva. https://www. who.int/ncd_surveillance/en/steps_framework_dec03.pdf

13. Whelton PK, Carey RM, Aronow WS, Casey DE, Collins KJ, Dennison Himmelfarb C, DePalma SM, Gidding S, Jamerson KA, Jones DW, MacLaughlin EJ, Muntner P, Ovbiagele B, Smith SC, Spencer CC, Stafford RS, Taler SJ, Thomas RJ, Williams KA, Williamson JD, Wright JT (2017) 2017 ACC/AHA/ AAPA/ABC/ACPM/AGS/APhA/ASH/ASPC/NMA/PCNA guideline for the prevention, detection, evaluation, and management of high blood pressure in adults. A Report of the American College of Cardiology/American Heart Association Task Force on Clinical Practice Guidelines. https://doi.org/10.1161/ hyp.0000000000000065

14. Chobanian AV, Bakris GL, Black HR et al (2003) The seventh report of the joint national committee on prevention, detection, evaluation, and treatment of high blood pressure: the JNC 7 report. JAMA 289(19):2560-2571. https://doi.org/10.1001/ jama.289.19.2560

15. Tanaka T, Okamura T, Miura K, Kadowaki T, Ueshima H, Nakagawa $\mathrm{H}$, Hashimoto T (2002) A simple method to estimate populational $24 \mathrm{~h}$ urinary sodium and potassium excretion using a casual urine specimen. J Hum Hypertens 16(2):97-103. https:// doi.org/10.1038/sj.jhh.1001307

16. Rezaei S, Mahmoudi Z, Sheidaei A, Aryan Z, Mahmoudi N, Gohari K, Yoosefi M, Hajipour MJ, Dilmaghani-Marand A, Soleimanzadehkhayat M, Gholami A, Mirab Samiee S, Moradi G, Larijani B, Farzadfar F (2018) Salt intake among Iranian population: the first national report on salt intake in Iran. J Hypertens. https:// doi.org/10.1097/hjh.0000000000001836

17. Armstrong T, Bull F (2006) Development of the World Health Organization Global Physical Activity Questionnaire (GPAQ). 
J Public Health 14(2):66-70. https://doi.org/10.1007/s1038 9-006-0024-x

18. Ando K, Fujita T (2012) Pathophysiology of salt sensitivity hypertension. Ann Med 44(Suppl 1):S119-S126. https://doi. org/10.3109/07853890.2012.671538

19. Sharma S, McFann K, Chonchol M, Kendrick J (2014) Dietary sodium and potassium intake is not associated with elevated blood pressure in US adults with no prior history of hypertension. J Clin Hypertens (Greenwich) 16(6):418-423. https://doi.org/10.1111/ jch. 12312

20. McLean RM (2014) Measuring population sodium intake: a review of methods. Nutrients 6(11):4651-4662. https://doi. org/10.3390/nu6114651

21. World Health Organization (2016) The SHAKE technical package for salt reduction. World Health Organization, Geneva. https ://apps.who.int/iris/bitstream/handle/10665/250135/9789241511 346-eng.pdf;jsessionid=A57688BEFD3F9F9F732998273595E4 68 ? sequence $=1$

22. He FJ, Brinsden HC, MacGregor GA (2013) Salt reduction in the United Kingdom: a successful experiment in public health. J Hum Hypertens 28:345. https://doi.org/10.1038/jhh.2013.105

23. Okuda N, Stamler J, Brown IJ, Ueshima H, Miura K, Okayama A, Saitoh S, Nakagawa H, Sakata K, Yoshita K, Zhao L, Elliott $P$ (2014) Individual efforts to reduce salt intake in China, Japan, UK, USA: what did people achieve? The INTERMAP population study. J Hypertens 32(12):2385-2392. https://doi.org/10.1097/ hjh.0000000000000341

24. Organization WH (2015) Report on the technical consultation on salt and fat reduction strategies in the Eastern Mediterranean
Region, Tunis, Tunisia 30-31 March 2015. World Health Organization. Regional Office for the Eastern Mediterranean

25. Strohm D, Boeing H, Leschik-Bonnet E, Heseker H, ArensAzevêdo U, Bechthold A, Knorpp L, Kroke A (2016) Salt intake in Germany, health consequences, and resulting recommendations for action. Ernaehrungs Umschau 63:62-70

26. Kloss L, Meyer JD, Graeve L, Vetter W (2015) Sodium intake and its reduction by food reformulation in the European Union-a review. NFS J 1:9-19. https://doi.org/10.1016/j.nfs.2015.03.001

27. Lee HS, Duffey KJ, Popkin BM (2012) Sodium and potassium intake patterns and trends in South Korea. J Hum Hypertens 27:298. https://doi.org/10.1038/jhh.2012.43 https://www.natur e.com/articles/jhh201243\#supplementary-information

28. Hyseni L, Elliot-Green A, Lloyd-Williams F, Kypridemos C, O'Flaherty M, McGill R, Orton L, Bromley H, Cappuccio FP, Capewell S (2017) Systematic review of dietary salt reduction policies: evidence for an effectiveness hierarchy? PLoS One 12(5):e0177535. https://doi.org/10.1371/journal.pone.0177535

29. McLaren L, Sumar N, Barberio AM, Trieu K, Lorenzetti DL, Tarasuk V, Webster J, Campbell NR (2016) Population-level interventions in government jurisdictions for dietary sodium reduction. Cochrane Database Syst Rev 9:Cd010166. https://doi. org/10.1002/14651858.cd010166.pub2

30. Rezaei S, Gholami A (2018) Letter to the editor about "Diet and primary prevention of stroke: systematic review and dietary recommendations by the ad hoc Working Group of the Italian Society of Human Nutrition". Nutr Metab Cardiovasc Dis. https://doi. org/10.1016/j.numecd.2018.04.007

\section{Affiliations}

\section{Ali Gholami ${ }^{1,2,3}$. Shahabeddin Rezaei ${ }^{4,5}$. Leila Moosavi Jahromi ${ }^{6} \cdot$ Hamid Reza Baradaran $^{3,7} \cdot$ Ali Ghanbari $^{4,8}$. Shirin Djalalinia ${ }^{4,9} \cdot$ Nazila Rezaei $^{4} \cdot$ Shohreh Naderimagham ${ }^{4} \cdot$ Mitra Modirian $^{4} \cdot$ Negar Mahmoudi $^{4}$. Zohreh Mahmoudi ${ }^{4,10} \cdot$ Mohammad Javad Hajipour $^{4,11} \cdot$ Ahmad kousha $^{12} \cdot$ Siamak Mirab Samiee $^{13}$. Farshad Farzadfar ${ }^{4,10}$ (1)}

1 Noncommunicable Diseases Research Center, Neyshabur University of Medical Sciences, Neyshabur, Iran

2 Department of Epidemiology and Biostatistics, School of Public Health, Neyshabur University of Medical Sciences, Neyshabur, Iran

3 Department of Epidemiology, School of Public Health, Iran University of Medical Sciences, Tehran, Iran

4 Non-Communicable Diseases Research Center, Endocrinology and Metabolism Population Sciences Institute, Tehran University of Medical Sciences, Tehran, Iran

5 Students' Scientific Research Center, Tehran University of Medical Sciences, Tehran, Iran

6 Health Management and Economics Research Center, Iran University of Medical Sciences, Tehran, Iran

7 Ageing Clinical and Experimental Research Team, Institute of Applied Health Sciences, School of Medicine, Medical Sciences and Nutrition University of Aberdeen, Aberdeen, UK
8 Department of Epidemiology and Biostatistics, School of Public Health, Tehran University of Medical Sciences, Tehran, Iran

9 Deputy of Research and Technology, Ministry of Health and Medical Education, Tehran, Iran

10 Endocrinology and Metabolism Research Center, Endocrinology and Metabolism Clinical Sciences Institute, Tehran University of Medical Sciences, Tehran, Iran

11 Persian Gulf Marine Biotechnology Research Center, The Persian Gulf Biomedical Sciences Research Institute, Bushehr University of Medical Sciences, Bushehr, Iran

12 Department of Health Education and Promotion, Faculty of Health Sciences, Tabriz University of Medical Sciences, Tabriz, Iran

13 Reference Health Laboratory, Ministry of Health and Medical Education, Tehran, Iran 